\title{
End-to-end Outage Probability Minimization in OFDM Based Linear Multi-Hop Networks
}

\author{
Xiaolu Zhang ${ }^{1}$, Meixia $\mathrm{Tao}^{1,2}$, Wenhua Jiao ${ }^{3}$, Chun Sum $\mathrm{Ng}^{1}$ \\ ${ }^{1}$ Dept. of Electrical \& Computer Engineering, National University of Singapore, Singapore 117576 \\ ${ }^{2}$ Dept. of Electronic Engineering, Shanghai Jiao Tong University, Shanghai, China 200240 \\ ${ }^{3}$ Bell Lab Research China (BLRC), Alcatel-Lucent, Beijing, China 100080
}

Emails: zhangxiaolu@nus.edu.sg; mxtao@ieee.org; wjiao@alcatel-lucent.com; elengcs@nus.edu.sg

\begin{abstract}
This paper is concerned with the end-to-end transmission outage in an OFDM based wireless multi-hop network. The network consists of a source node, a destination node, and a set of serial relay nodes. Transmission outage occurs when the end-to-end transmission rate is less than a target rate. Our goal is to find an optimal power and time control policy to minimize the outage probability at a target end-to-end data rate under a long-term total power constraint. The problem is solved in two steps. First, for any given channel realization, we derive the minimum total power required to meet the target rate by varying the transmission power and time allocated to each hop. This is formulated as a convex optimization problem and is solved efficiently. We then compare the required minimum total power for each channel realization with a power threshold. If the threshold is exceeded, the transmission is turned off. The optimal threshold is chosen to meet the long-term total power constraint. Two suboptimal power and time control policies are also presented and compared with the optimal policy by simulation.
\end{abstract}

\section{INTRODUCTION}

Point-to-multi-point (PMP) and mesh networks are two of the most promising topologies in future wireless networks. Under these two architectures, a source is allowed to communicate with a destination under the help of multiple relay nodes. The concept of relaying has been widely adopted in wireless networks such as next generation cellular networks, broadband wireless metropolitan area networks (WMANs) and wireless local area networks (WLANs). On the other hand, Orthogonal Frequency Division Multiplexing (OFDM) is a promising physical layer technique for broadband wireless communications. It divides the broadband channel into orthogonal narrowband subcarriers to eliminate the inter-symbolinterference. OFDM has been adopted in many wireless systems, e.g., IEEE 802.16. OFDM based multi-hop wireless networks have received a lot of attention recently. In particular, this OFDM-based relaying architecture has been proposed by current wireless standard IEEE 802.16j [1]. To simplify the resource allocation to the source-to-relay and relay-todestination links, a time-division multi-hop relay scheme is assumed as shown in Fig. 1. Time is divided into frames of multiple time slots. At the beginning of each frame, it needs to determine the transmission parameters including the transmission power on each subcarrier over each hop and the transmission time over each hop.
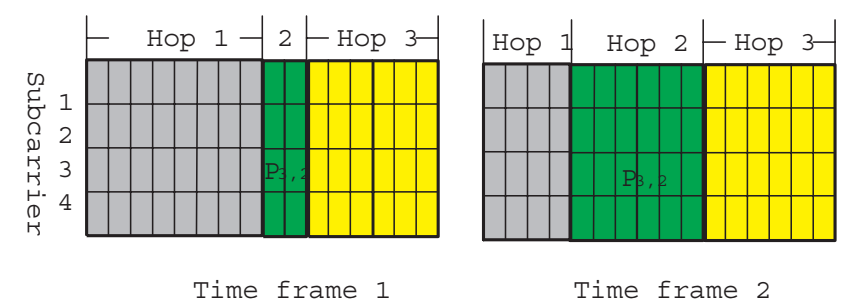

Fig. 1. Time-division OFDM based relaying system with 4 subcarriers and 3 hops

Previous work on resource allocation for relay networks is found in [2]-[5]. Yao et al. in [2] consider a system with a source node, a destination node, and a single relay and compare the energy required for transmitting one information bit in different relay schemes. Authors in [3] and [4] studied efficient scheduling and routing schemes in one-dimensional multi-hop wireless networks. It is assumed in [2]-[4] that the point-to-point links are frequency-flat fading channels and the system has a fixed short-term power constraint. In [5], Oyman et al. introduce two different transmission strategies over multiple hops, and show merits of multi-hop relaying in cellular mesh networks. Based on the framework proposed in [5] and [6], the authors in [7] studied the resource allocation problem in an OFDM based multi-hop network to maximize the end-to-end average transmission rate under a long-term total power constraint. However, the end-to-end average rate can only be obtained at the expense of large delay.

Few existing works consider transmission outage in the multi-hop wireless network, which is an important performance metric for many real-time services. An outage is an event where the actual transmission rate is below a prescribed transmission rate ( [8] and [9]). Outage probability can be viewed as the fraction of time that a codeword is decoded wrongly. For a given average power constraint, it may not be able to support the target rate all the time. Thus, transmission outage is inevitable under severe fading condition.

In this paper, we study the power and time control to minimize the end-to-end outage probability in a one-dimensional multi-hop network under an average transmission power constraint. The end-to-end outage is defined as the event when the end-to-end transmission rate is lower than a target rate. For any 

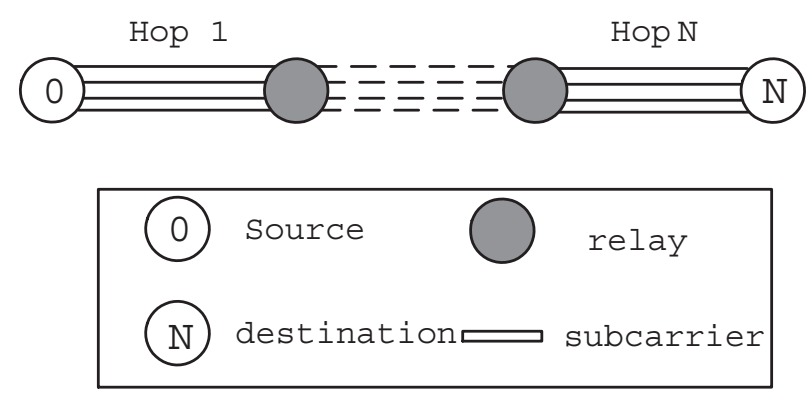

Fig. 2. Illustration of linear multi-hop networks

given channel realization, we first derive the minimum total power required to meet a target rate. The transmission onoff is then determined by comparing the required minimum total power with a threshold. The threshold takes the value so that the long-term total power constraint is satisfied. Numerical results show that a significant power saving can be achieved by the proposed optimal power and time allocation compared with uniform power and time allocation under the same end-to-end outage probability. It is also shown that multi-hop transmission greatly reduces the outage probability compared with singlehop transmission if the target rate is small.

The remainder of this paper is organized as follows. In Section II, the system model and problem formulation are presented. The adaptive power and time allocation to minimize the end-to-end outage probability is derived in Section III. Numerical results are given in Section IV. Finally we conclude this paper in Section V.

\section{System Model AND Problem Formulation}

Consider a linear multi-hop wireless network shown in Fig. 2. The source node $R_{0}$ communicates with destination $R_{N}$ by routing its data through multiple relay nodes $R_{n}(n=$ $1, \ldots, N-1)$. The hop between node $R_{n-1}$ and $R_{n}$ is indexed by $n$, and the set of hops is denoted by $\mathcal{N}$. We focus on timedivision based relaying. In each time frame, the message from the source is sequentially relayed at each hop using decodeand-forward scheme [10]. Over each hop, OFDM with $K$ subcarriers is used as the transmission technology. The set of subcarriers is denoted by $\mathcal{K}$. We assume that the channel between two nodes is a block fading Gaussian channel, and the channel gain on subcarrier $k$ over hop $n$, denoted as $g_{k, n}$, is random but constant during the entire time frame.

Denote the transmission power on subcarrier $k$ over hop $n$ by $p_{k, n}$ and let the long-term total transmission power be limited by $P$. Let $\rho_{n}{ }^{1}$ represent the amount of time that hop $n$ is scheduled to transmit during a time frame with period $T$. Without loss of generality, the time frame duration $T$ is normalized to one. Then the transmission rate (Nat/OFDM symbol) in a time frame achieved by subcarrier $k$ over hop $n$ is

$$
r_{k, n}=\rho_{n} \ln \left(1+\frac{g_{k, n} p_{k, n}}{\Gamma N_{0}}\right),
$$

\footnotetext{
${ }^{1}$ We assume the number of time slots in a time frame is large enough so that $\rho_{n}$ can take arbitrary value between 0 and $T$
}

where $N_{0}$ is the noise power, and $\Gamma$ is the signal-to-noise ratio (SNR) gap [11]. For notation brevity, in the remaining part of this paper, we redefine $g_{k, n}$ as $g_{k, n}:=g_{k, n} / \Gamma N_{0}$. The total transmission rate over hop $n$ is denoted as $r_{n}=$ $\sum_{k \in \mathcal{K}} r_{k, n}$. The end-to-end transmission rate is defined as the minimum of transmission rates over the $N$ hops, as in [5] and [6]. Mathematically, $r=\min _{n \in \mathcal{N}} r_{n}$.

If the end-to-end transmission rate $r$ is less than a target transmission rate $R$, then an outage is said to occur. Given the global channel state information (CSI), our goal is to find an optimal power and time allocation to minimize the outage probability for a given target transmission rate subject to a long-term total power constraint. Namely,

$$
\begin{aligned}
\text { P1: } \min _{\mathbf{p}, \boldsymbol{\rho}} & \mathbb{E}\left\{\mathcal{I}_{F}[r(\mathbf{g}, \boldsymbol{\rho}, \mathbf{p})<R]\right\} \\
\text { s.t. } & \mathbb{E}\left[\sum_{n \in \mathcal{N}} \rho_{n}(\mathbf{g})\left(\sum_{k \in \mathcal{K}} p_{k, n}(\mathbf{g})\right)\right] \leq P \\
& \sum_{n \in \mathcal{N}} \rho_{n}(\mathbf{g})=1,
\end{aligned}
$$

where $\mathbf{g}=\left\{g_{k, n}\right\}, \boldsymbol{\rho}=\left\{\rho_{n}\right\}, \mathbf{p}=\left\{p_{k, n}\right\}, \mathcal{I}_{F}$ is an indicator function and $\mathbb{E}(x)$ denotes the expectation of $x$ over the joint distribution of $\mathrm{g}$.

\section{AdAPTIVE POWER AND TIME ALLOCATION (APTA)}

The minimum outage probability problem under the longterm power constraint can be generally solved in two steps as proposed in [8]. First, for each global channel state $\mathrm{g}$, the short-term minimum total power $P_{\min }(\mathrm{g}, R)$ required to guarantee the target end-to-end transmission rate $R$ is to be determined. The second step then determines a threshold to control the transmission on-off subject to a long-term power constraint.

\section{A. Short-Term Power Minimization}

In this subsection, we shall find the optimal time sharing fraction $\rho_{n}(\forall n \in \mathcal{N})$ and the optimal power allocation $p_{k, n}(\forall n \in \mathcal{N}, k \in \mathcal{K})$ to minimize the short-term total power needed to achieve a target end-to-end transmission rate $R$. Mathematically, it can be formulated as

$$
\text { P2: } \begin{aligned}
p_{\min }(\mathbf{g})= & \min _{\left\{\rho_{n}, p_{k, n}\right\}} \sum_{n \in \mathcal{N}} \rho_{n}(\mathbf{g})\left(\sum_{k \in \mathcal{K}} p_{k, n}(\mathbf{g})\right) \\
\text { s.t. } \quad & r(\mathbf{g}, \boldsymbol{\rho}, \mathbf{p}) \geq R \\
& \sum_{n \in \mathcal{N}} \rho_{n}=1 .
\end{aligned}
$$

Unfortunately, the term $r(\mathbf{g}, \boldsymbol{\rho}, \mathbf{p})$ in constraint (5) is not concave in $\boldsymbol{\rho}$ and $\mathbf{p}$. To make the problem $\mathbf{P 2}$ more tractable, we introduce a new variable $s_{k, n}$ defined as $s_{k, n}:=\rho_{n} p_{k, n}$. In addition, constraint (5) can be rewritten as $N$ sub-constraints since $r=\min _{n}\left(r_{n}\right)$. By doing so, problem $\mathbf{P 2}$ can be transformed into a new problem with optimization variables 
$\rho_{n}(\forall n \in \mathcal{N})$ and $s_{k, n}(\forall n \in \mathcal{N}, k \in \mathcal{K}):$

$$
\text { P3: } \begin{aligned}
\min _{\left\{\rho_{n}, s_{k, n}\right\}} & \sum_{n \in \mathcal{N}} \sum_{k \in \mathcal{K}} s_{k, n} \\
\text { s.t. } \quad & \rho_{n} \sum_{k \in \mathcal{K}} \ln \left(1+\frac{g_{k, n} s_{k, n}}{\rho_{n}}\right) \geq R, \forall n \\
& \sum_{n \in \mathcal{N}} \rho_{n}=1 .
\end{aligned}
$$

Since its Hessian matrix is negative semidefinite, the function $\rho_{n} \ln \left(1+g_{k, n} s_{k, n} / \rho_{n}\right)$ is concave in $\rho_{n}$ and $s_{k, n}$. Therefore, problem $\mathbf{P} 3$ is a convex optimization problem and there exists a unique optimal solution.

To observe the structure of the optimal solution, we write the Lagrangian of Problem P3 as follows:

$$
\begin{aligned}
& J\left(\left\{\rho_{n}\right\},\left\{s_{k, n}\right\},\left\{\lambda_{n}\right\}, \beta\right)=\sum_{n \in \mathcal{N}} \sum_{k \in \mathcal{K}} s_{k, n}-\beta\left(1-\sum_{n \in \mathcal{N}} \rho_{n}\right) \\
& -\sum_{n \in \mathcal{N}} \lambda_{n}\left[\rho_{n} \sum_{k \in \mathcal{K}} \ln \left(1+\frac{g_{k, n} s_{k, n}}{\rho_{n}}\right)-R\right],
\end{aligned}
$$

where $\lambda_{n} \geq 0(n \in \mathcal{N})$ and $\beta \geq 0$ are the Lagrange multipliers for the constraints (7) and (8), respectively. If $\left\{\rho_{n}^{*}\right\}$ and $\left\{s_{k, n}^{*}\right\}$ are the optimal solution of $\mathbf{P 3}$, they should satisfy the Karush-Kuhn-Tucker (KKT) conditions [12], which are necessary and sufficient for the optimality.

Taking the derivative of the Lagrangian with respect to $s_{k, n}$ and equating it to zero, we obtain the optimal power distribution $\left\{p_{k, n}^{*}\right\}$, which has a water-filling structure given by

$$
p_{k, n}^{*}=\frac{s_{k, n}^{*}}{\rho_{n}^{*}}=\left(\lambda_{n}-\frac{1}{g_{k, n}}\right)^{+}, \forall k \in \mathcal{K}, n \in \mathcal{N},
$$

where $(x)^{+} \triangleq \max (0, x)$, and $\lambda_{n}$ can be regarded as the water-filling level of hop $n$.

Define $\mathcal{K}_{n}$ as the set of active subcarriers $\left(p_{k, n}>0\right)$ over hop $n$, and $k_{n}$ as the size of the set $\mathcal{K}_{n}$. Substituting (9) into (7) and applying KKT conditions, we can obtain the closedform expression for $\rho_{n}^{*}$ in $\lambda_{n}$ and $\beta$ :

$$
\rho_{n}^{*}=h_{n}\left(\mathbf{g}, \lambda_{n}\right) \triangleq \frac{R}{\sum_{k \in \mathcal{K}_{n}} \ln g_{k, n}+k_{n} \ln \lambda_{n}}, \forall n \in \mathcal{N} .
$$

From (10), it can be shown that $\rho_{n}^{*}$ is decreasing in $\lambda_{n}$. By taking the derivative of the Lagrangian with respect to $\rho_{n}$, letting it equal to 0 , and substituting (9) into the result, it is also obtained that

$$
\begin{aligned}
\beta=f_{n}\left(\mathbf{g}, \lambda_{n}\right) \triangleq & \lambda_{n}\left(\sum_{k \in \mathcal{K}_{n}} \ln g_{k, n}+k_{n} \ln \lambda_{n}-k_{n}\right) \\
& +\sum_{k \in \mathcal{K}_{n}} \frac{1}{g_{k, n}}, \forall n \in \mathcal{N} .
\end{aligned}
$$

It is seen from (11) that finding the optimal water-filling level $\left\{\lambda_{n}\right\}$ for a given $\beta$ are $N$ independent problems. It can be proven that $f_{n}\left(\mathrm{~g}, \lambda_{n}\right)$ is an increasing function of $\lambda_{n}$ in the region $\left[\min _{k}\left(1 / g_{k, n}\right),+\infty\right]$. Hence, the inverse function, $f_{n}^{-1}(\mathrm{~g}, \beta)$, exists and is an increasing function of $\beta$ in that region. Therefore, the exact value of $\lambda_{n}$ can be obtained numerically using binary search when the upper bound is known.

Substituting $\lambda_{n}=f_{n}^{-1}(\mathbf{g}, \beta)$ in (10), we can express $\rho_{n}$ as $\rho_{n}=h_{n}\left(\mathbf{g}, f_{n}^{-1}(\mathbf{g}, \beta)\right)$. Since $\rho_{n}$ is decreasing in $\lambda_{n}$ and $\lambda_{n}$ is increasing in $\beta$, we have that $\rho_{n}$ is decreasing in $\beta$. Therefore, the optimal $\beta$ can also be obtained via binary search from the constraint $\sum_{n \in \mathcal{N}} \rho_{n}=1$. Hence, the optimal solution $\left\{\rho_{n}^{*}, s_{k, n}^{*}\right\}$ of $\mathbf{P 3}$ can be obtained through two-nested binary search.

Consider a special case when the target rate is sufficiently large. It can be shown that the water-filling levels $\left\{\lambda_{n}\right\}$ tend to be the same for all $n$, and all the subcarriers on each hop become active, i.e., $k_{n}=K(\forall n \in \mathcal{N})$. Then equation (10) indicates that the hops with low geometric mean of channel gains over the subcarriers should be assigned with longer transmission time. Moreover, by summing (9) over the $K$ subcarriers, it is seen that the hops with low harmonic mean of channel gains should be given lower transmission power. In our future work, we shall investigate a suboptimal solution with low computational complexity by forcing equal waterfilling levels at all hops.

\section{B. Long-Term Power Threshold Determination}

We have discussed the short-term total transmission power minimization. If the transmission is on for every possible channel realization, the long term power constraint may be violated. Similar to the single user case [8], the optimal power allocated to all hops for $\mathbf{P 1}$ with a long term constraint must have the following structure,

$$
p(\mathbf{g})=\left\{\begin{array}{ll}
p_{\min }(\mathbf{g}) & \text { with probability } w(\mathbf{g}) \\
0 & \text { with probability } 1-w(\mathbf{g})
\end{array},\right.
$$

where $p_{\min }(\mathrm{g})$ is defined in (4). Thus, the outage probability is $\mathbb{E}\left\{\mathcal{I}_{F}[r(\mathbf{g}, \boldsymbol{\rho}, \mathbf{p})<R]\right\}=\mathbb{E}[1-w(\mathbf{g})]$. Then solving P1 is equivalent to finding the optimal weighting function $w(\mathrm{~g})$ to the following problem,

$$
\begin{aligned}
\min _{w(\mathbf{g})} & \mathbb{E}[1-w(\mathbf{g})] \\
\text { s.t. } & 0 \leq w(\mathbf{g}) \leq 1 \\
& \mathbb{E}\left[p_{\min }(\mathbf{g}) w(\mathbf{g})\right]=P .
\end{aligned}
$$

According to the result of [8, Lemma 3], the optimal weighting function has the form

$$
w^{*}(\mathbf{g})=\left\{\begin{array}{ll}
1 & \text { for } p_{\min }(\mathbf{g})<s^{*} \\
w_{0} & \text { for } p_{\min }(\mathbf{g})=s^{*} \\
0 & \text { for } p_{\min }(\mathbf{g})>s^{*}
\end{array} .\right.
$$

The power threshold $s^{*}$ is given by

$$
s^{*}=\sup \{s: \mathcal{P}(s)<P\},
$$

and $w_{0}$ is given by

$$
w_{0}=\frac{P-\mathcal{P}\left(s^{*}\right)}{\overline{\mathcal{P}}\left(s^{*}\right)-\mathcal{P}\left(s^{*}\right)},
$$


where the region $\mathcal{R}(s)$ and $\overline{\mathcal{R}}(s)$ are defined as

$$
\mathcal{R}(s)=\left\{\mathbf{g}: p_{\min }(\mathbf{g})<s\right\}, \overline{\mathcal{R}}(s)=\left\{\mathbf{g}: p_{\min }(\mathbf{g}) \leq s\right\},
$$

and the corresponding average power over the two sets are:

$$
\mathcal{P}(s)=\mathbb{E}_{\mathbf{g} \in \mathcal{R}(s)}\left[p_{\min }(\mathbf{g})\right], \overline{\mathcal{P}}(s)=\mathbb{E}_{\mathbf{g} \in \overline{\mathcal{R}}(s)}\left[p_{\min }(\mathbf{g})\right] .
$$

The resulting minimum outage probability is given by

$$
P_{\text {out }}=1-\operatorname{Prob}\left\{\mathbf{g} \in \mathcal{R}\left(s^{*}\right)\right\}-w_{0} \operatorname{Prob}\left\{p_{\min }(\mathbf{g})=s\right\} .
$$

From (12) and (13), we see that if the minimum total power $p_{\text {min }}(\mathbf{g})$ at channel state $\mathbf{g}$ required to support the target transmission rate is beyond the threshold $s^{*}$, transmission is turned off. Otherwise if the required power is less than the threshold, the transmission follows the minimum transmission power strategy derived from Section III-A.

The value of $s^{*}$ can be computed in advance if the fading statistics are known. Otherwise, the threshold can be estimated iteratively using fading samples. Since the channel is assumed to be ergodic, the ensemble average transmission power is equal to the time average

$$
\mathbb{E}_{\mathbf{g} \in \mathcal{R}(s)}\left[p_{\min }(\mathbf{g})\right]=\lim _{t \rightarrow \infty} \frac{1}{t} \sum_{i=1}^{t} \hat{p}(i),
$$

where $\hat{p}_{(i)}$ represents the actual transmission power at time frame $i$. The threshold at time frame $(t+1)$ can then be adjusted in the opposite direction of $P-\frac{1}{t} \sum_{i=1}^{t} \hat{p}_{(i)}$ as

$$
s^{*}(t+1)=s^{*}(t)\left[1+\epsilon\left(P-\frac{1}{t} \sum_{i=1}^{t} \hat{p}_{(i)}\right)\right] .
$$

\section{NUMERICAL RESULTS}

In this section, we present some numerical results to illustrate the performance of the proposed adaptive power and time allocation for OFDM based linear networks. The proposed algorithm is compared with two suboptimal algorithms:

Adaptive power control with fixed time-sharing fraction (APFT): APFT is a suboptimal solution of APTA by assuming equal time allocation with $\rho_{n}=1 / N$, for all $n$. The waterlevel $\lambda_{n}$ in this suboptimal algorithm is computed locally at each hop $n$ using (10) by letting $\rho_{n}^{*}=1 / N$. Then, substituting the resulting $\lambda_{n}$ into (9), we obtain the power control. The long-term power threshold is still obtained using the same method discussed in Section III-B. Compared with APTA, APFT considerably reduces the computational complexity as well as signaling overhead.

Uniform power and time allocation (UPTA): The power is distributed equally among all hops and all the subcarriers, and the time is allocated equally to each hop. This algorithm is proposed for benchmark purpose. Its main disadvantage is that any one of hops which suffers from bad channel condition may become the bottleneck of the whole link.

We consider an $N$-hop linear wireless network. The acceptable BER is chosen to be $10^{-5}$, which corresponds to $8.2 \mathrm{~dB}$ SNR gap. We fix the bandwidth to be $1 \mathrm{MHz}$ and the endto-end distance to be $1 \mathrm{~km}$. The relays are equally spaced. In

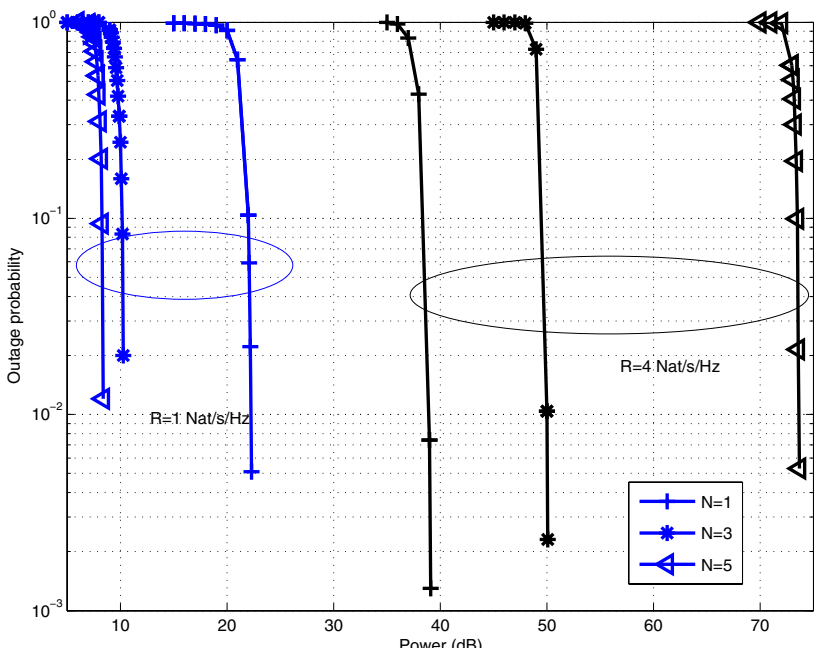

Fig. 3. End-to-end outage probability vs. average total transmission power under APTA when $K=16$

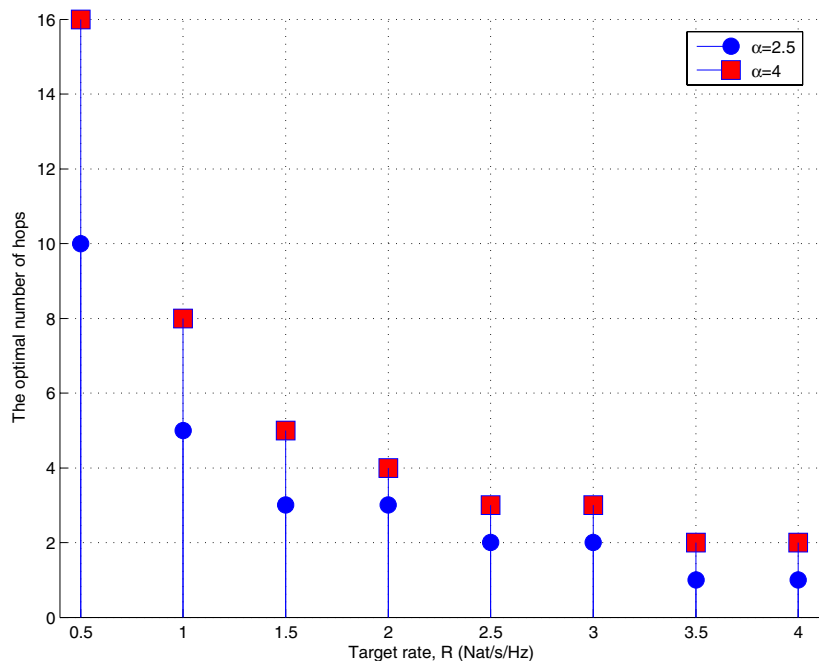

Fig. 4. Optimal number of hops vs. target rate under APTA when $\alpha=2.5$ and 4

all simulations, we adopted the Stanford University Interim (SUI)-3 channel model with a central frequency at around $3.4 \mathrm{GHz}$, which is suitable for fixed broadband wireless access channel environments [13]. The SUI-3 channel is a 3-tap channel with $0.305 \mu$ s root-mean-square (rms) delay spread. The coherence bandwidth is approximately $65 \mathrm{KHz}$. Hence, the number of subcarriers $\mathrm{K}$ should be greater than 15.2 so that the subcarrier bandwidth is small enough to experience the flat fading. Here we choose $K=16$. In each simulation, $10^{4}$ time frames are used to estimate the outage probability.

Fig. 3 shows the end-to-end outage probability versus the long-term total transmission power for $R=1$ and $4 \mathrm{Nats} / \mathrm{s} / \mathrm{Hz}$ using APTA when $N$ varies in the set of $\{1,3,5\}$. From the figure, it is shown that multi-hop transmission can dramatically reduce the total power consumption when the target transmission rate is low (e.g., $R=1$ ) whereas it is better to send data directly to the destination if the target transmission rate is high (e.g., $R=4$ ). That can be explained by the following fact. As the number of hops increases, the path loss attenuation on each hop reduces. But the transmission time spent at each hop also 


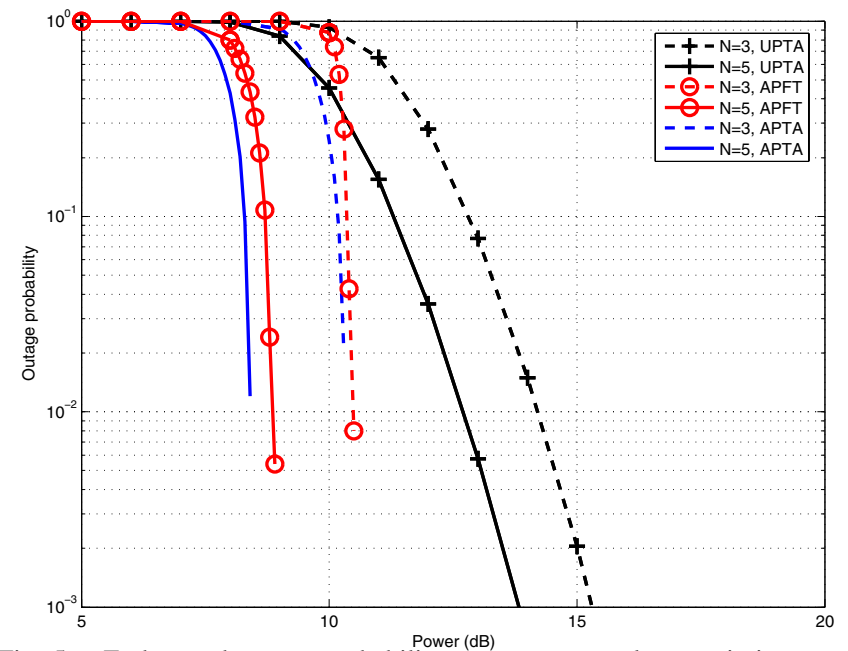

Fig. 5. End-to-end outage probability VS. average total transmission power under APTA, APFT and UPTA when $K=16$ and $R=1 \mathrm{Nat} / \mathrm{s} / \mathrm{Hz}$

reduces since the total frame length is fixed. It is observed from (1) that the transmission rate is linear in transmission time and concave in channel gain. Hence, when target transmission rate increases, the loss due to the reduction of transmission time goes beyond the benefit from the reduction of path loss attenuation.

Fig. 4 shows the relationship between the optimal number of hops (in the sense of minimizing required power consumption) and the target transmission rate, when the outage probability is fixed to $1 \%$. Two different path loss exponents, $\alpha=2.5$ and 4 , are considered. It is observed that the optimal number of hops is roughly proportional to the inverse of $R$, and it increases linearly in $\alpha$. A similar trend is shown in [14] where a special case, frequency-flat fading channel and fixed short-term total power constraint, is considered.

Fig. 5 compares the end-to-end outage probabilities of APTA, APFT and UPTA when $R=1 \mathrm{Nat} / \mathrm{s} / \mathrm{Hz}$. There is a considerable reduction in total transmit power by using both proposed APTA and APFT compared with the uniform resource allocation. In particular, a power saving gain of around $4 \mathrm{~dB}$ is achieved at outage probability of $1 \%$ with $N=3$ hops. Recall that UPTA has a fixed short-term total power consumption while both APTA and APFT use adaptive transmission power with respect to instantaneous channel condition subject to a long-term power constraint. Thus, a significant gain over UPTA can be achieved by turning off the transmission when the channel is in deep fade. It is also observed that the performance gap between APTA and APFT is very small. Therefore, it suffices to use APFT in practical systems because of its low computational complexity and signalling overhead.

Overall, the numerical results suggest that multi-hop transmission is favorable when the target rate is low, whereas a direct transmission from source to destination is preferred if the target rate is large. Moreover, APFT is an effective power and time control policy in practice since it has a similar performance with the optimal APTA scheme and yet is much less complex.

\section{CONCLUSiON}

In this work, we consider an adaptive power and time allocation for OFDM based linear multi-hop networks for end-to-end outage probability minimization. We first derive the minimum short-term total power required to meet the target transmission rate. Then, the transmission on-off is determined by comparing the required minimum total power with a threshold, which is selected to achieve a long-term total power constraint. Numerical results demonstrate the outage probability of the proposed optimal APTA algorithm and suboptimal APFT algorithm, in comparison with the uniform power and time allocation. It is shown that APTA has a significant performance gain over UPTA, but the gap between APTA and APFT is small. We also find that the optimal number of hops decreases when the target end-to-end transmission rate increases, and that singlehop transmission should be preferred if the target rate is high enough.

\section{REFERENCES}

[1] R. Pabst, B. Walke, D. Schultz, P. Herhold, H. Yanikomeroglu, S. Mukherjee, H. Viswanathan, M. Lott, W. Zirwas, M. Dohler, H. Aghvami, D. Falconer, and G. Fettweis, "Relay-based deployment concepts for wireless and mobile broadband radio," IEEE Comm. Magazine, vol. 42, no. 9, pp. 80-89, 2004.

[2] Y. Yao, X. Cai, and G. Giannakis, "On energy efficiency and optimum resource allocation of relay transmissions in the low-power regime," IEEE Trans. Wirel. Commun., vol. 4, no. 6, pp. 2917 - 2927, Nov. 2005.

[3] M. Sikora, J. N. Laneman, M. Haenggi, J. Daniel J. Costello, and T. E. Fuja, "Bandwidth-and power-efficient routing in linear wireless networks," IEEE Trans. Inf. Theory, vol. 52, pp. 2624-1633, 2006.

[4] B. Radunovic and J.-Y. L. Boudec, "Joint scheduling, power control and routing in symmetric, one-dimensional, multi-hop wireless networks," in In WiOpt, France, March 2003.

[5] O. Oyman, J. N. Laneman, and S. Sandhu, "Multihop relaying for broadband wireless mesh networks: From theory to practice," IEEE Comm. Mag., vol. 45, no. 11, pp. 116-122, Nov. 2007.

[6] O. Oyman and S. Sandhu, "Non-ergodic power-bandwidth tradeoff in linear multi-hop networks," in Proc. ISIT, July 2006.

[7] X. Zhang, W. Jiao, and M. Tao, "End-to-end resource allocation in OFDM based linear multi-hop networks," in Proc. IEEE Infocom 2008, Phoenix, USA, April 2008.

[8] G. Caire, G. Taricco, and E. Biglieri, "Optimum power control over fading channels," IEEE Trans. Inf. Theory, vol. 45, no. 5, pp. 14681489, 1999.

[9] L. Li and A. Goldsmith, "Capacity and optimal resource allocation for fading broadcast channels: Part II: outage capacity," IEEE Trans. Info. Theory, vol. 47, no. 3, pp. 120-145, 2000.

[10] J. N. Laneman, D. N. C. Tse, and G. W. Wornell, "Cooperative diversity in wireless networks: efficient protocols and outage behavior," IEEE Trans. Inf. Theory, vol. 50, pp. 3062-3080, 2004.

[11] X. Qiu and K. Chawla, "On the performance of adaptive modulation in cellular systems," IEEE Trans. on Commun., vol. 47, no. 6, pp. 884-895, June 1999.

[12] S. Boyd and L. Vandenberghe, Convex Optimization. Cambridge, United Kingdom: Cambridge Univ. Press, 2004.

[13] V. Erceg, K. Hari, M. Smith, and D. Baum et al, "Channel models for fixed wireless applications," IEEE 802.16.3c-01/29r1, 23 Feb 2001.

[14] M. Sikora, J. N. Laneman, M. Haenggi, J. D. J. Costello, and T. E. Fuja, "On the optimum number of hops in linear ad hoc networks," in in Proc. IEEE Inform. Theory Workshop (ITW), San Antonio, Oct 2004, pp. $165-169$. 